\title{
Traffic Flow Theory for One Dimension and Formation of Shock Speed
}

\author{
Wah Wah Aung \\ Department of Engineering \\ Mathematics \\ Technological University \\ (Thanlyin) \\ Myanmar
}

\author{
Mya Thida Hlaing \\ Department of Engineering \\ Mathematics \\ Technological University \\ (Thanlyin) \\ Myanmar
}

\author{
Zaw Min Tun \\ Department of Engineering \\ Mathematics \\ West Yangon Technological \\ University (Hlaing Tharyar) \\ Myanmar
}

\begin{abstract}
This paper presents the necessary background on traffic flow theory and the existing macroscopic mathematical models for single-lane, one-dimensional space traffic flow. The derivation of traffic flow theory based on conservation of mass and the relationships between velocity and density are presented. The exact and weak solutions to the scalar traffic flow Partial Differential Equation, and the shock wave, rarefaction wave, and the admissibility of a solution are considered.
\end{abstract}

Keywords: Conservation Laws, Boundary Conditions, Traffic Flow, Weak Solutions. Car Following Model.

\section{INTRODUCTION}

In traffic flow problem, nonlinear hyperbolic conservation laws have been studied for many years. Traffic flows are classified according to traffic conditions, roadway conditions and traffic network structures. Traffic flows are considered inhomogeneous when the roadway has different parameters at different locations. A mathematical model for fluid dynamic flows on road networks is based on conservation laws. In this paper, we considered the first order traffic flows model. The first order model is based on the conservations of cars and is described by a single equation in conservation form. The main assumption of the car following models is that an individual car's motion only depends on the car ahead. As traffic jams display sharp discontinuities, there is a correspondence between traffic jams and shockwaves. We used the conservation laws model on the roads with time varing traffic distribution coefficients. In order to obtain a unique solution of the Riemann problem at junctions, we need to assume some rules, so we can construct solutions via wavfront tracking technique. To describe a road network as a finite collection of roads meeting at some junctions that play a key role, since the system at a junction is under-determined even after imposing the conservation of cars. Traffic models are represented by minimization of congestions, accidents, pollution, and the maximization of safety. Shock and rarefaction waves are the basic solutions to a Riemann problem for such a conservation law.

The paper is organized as follows. In section 2, the model for traffic flow on a road network is described. Section 3 deals with weak solutions, scalar Riemann problem and Numerical Method use for the traffic flow problem. Finally we discuss the shock formation.

\section{VELOCITY FIELD}

Consider a car moving along a highway. Since there is no passing and cars cannot move through each other, the order of the cars is preserved, although they can move at slightly different speeds. Let the velocity of car " $i$ " be $v_{i}$. If the $x$ axis coincides with the road and position of this car is $x_{i}(t)$ at time $\boldsymbol{t}$, then $v_{i}(t)=\frac{d x_{i}}{d t}, i=1,2, \ldots, N$

with $N$ cars there are different velocities, $v_{i}(t), i=1, . ., N$, each depending on time.

\section{TRAFFIC DENSITY}

Traffic density $\rho(x, t)$ is the average number of cars per unit length at the position $x$ and time $t$. The flow can be written as

$$
f(x, t)=\rho(x, t) v(x, t),
$$

where $\rho$ is the density of cars, $v$ is the mean velocity and $f$ is the traffic flow.

As the density increases, the velocity of cars diminishes. Thus we make the hypothesis that the velocity of cars at any point of the road is a strictly decreasing function of the density:

$$
v=v(\rho) .
$$

If there are no other cars on the highway, then the car would travel at the maximum speed $v_{\max }$,

$$
v(0)=v_{\max } \cdot
$$

$v_{\text {max }}$ is sometimes referred to as the "mean free speed" corresponding to the velocity cars would travel if they were free from interference from other cars. At a certain density cars stop before they touch to each other. The maximum density $\rho_{\max }$, usually corresponds to what is called bumperto-bumper traffic:

$$
v\left(\rho_{\max }\right)=0 .
$$

In our case the flux is the following 
$f(\rho)=v_{\max } \rho\left(1-\frac{\rho}{\rho_{\max }}\right)$,

where we set for simplicity $\rho_{\max }=1=v_{\max }$, thus it reads:

$$
f(\rho)=\rho(1-\rho) \text {. }
$$

\section{CAR FOLLOWING MODEL}

To derive the one-dimensional model, first assume cars cannot pass each other. Then the idea is that a car in onedimension can move and accelerate forward based on two parameters; the headway distance between the current car and the one in front, and their speed difference. Hence, it is called car following, where a car from behind follows the one in front, and this is the anisotropic property Suppose the $n^{\text {th }}$ car location is $x_{n}(t)$, then the nonlinear model is given by

$$
\ddot{x}_{n}(t)=c \frac{\dot{x}_{n}(t)-\dot{x}_{n-1}(t)}{x_{n}(t)-x_{n-1}(t)} .
$$

The acceleration of the current car $\ddot{x}_{n}(t)$ depends on the front car speed and location, $c$ is the sensitivity parameter. Integrating the above yields

$\dot{x}_{n}(t)=c \ln \left(x_{n}(t)-x_{n-1}(t)\right)+d_{n}$. Since by the definition of the density (number of cars per unit area)

$\frac{1}{\rho(x, t)}=x_{n}(t)-x_{n-1}(t)$,

and the integration constant $d_{n}$ is chosen such that at jam density $\rho_{\max }$, the velocity is zero. Then for steady-state we get

$$
v=-c \ln \frac{\rho}{\rho_{m}} .
$$

It can be seen that $\rho \rightarrow 0$ traffic densities are low, car speed is the maximum allowed speed, hence we can assume $v=v_{\text {max }}$, which is the maximum allowed speed.

\subsection{Traffic Flow}

The average number of cars passing per time unit is called the traffic flow $f=f(x, t)$. Suppose there is a road with cars moving with constant velocity $v_{0}$, and constant density $\rho_{0}$ such that the distance between the cars is also constant as shown in the Figure. 1(a). Now let an observer measure the number of cars per unit time $t$ that pass him (i.e. traffic flow $f$ ). In $t$ time, each car has moved $v_{0} t$ distance, and hence the number of cars that pass the observer in $t$ time is the number of cars in $v_{0} t$ distance, see in Figure. 1(b). Since the density $\rho_{0}$ is the number of cars per unit area and there is $v_{0} t$ distance, then the traffic flow is given by

$$
f=\rho_{0} v_{0} .
$$

This is the same equation as in the time varying case,

$$
f(\rho, v)=\rho(x, t) v(x, t) .
$$

To show this, we consider the number of cars that pass point $x=x_{0}$ in a very small time $\Delta t$. In this period of time the cars have not moved far and hence $\rho(x, t)$ and $v(x, t)$ can be approximated by their constant values at $x=x_{0}$ and $t=t_{0}$. Then, the number of cars passing the observer occupies a short distance, and they are approximately equal to, $\rho(x, t) v(x, t) \Delta t$ where the traffic flow is given by Equation 6 .

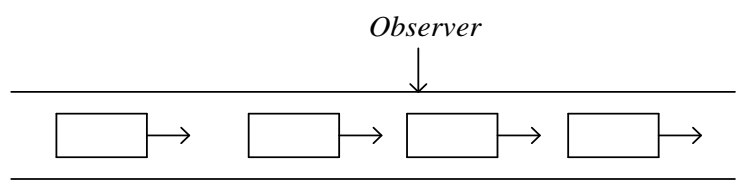

(a)

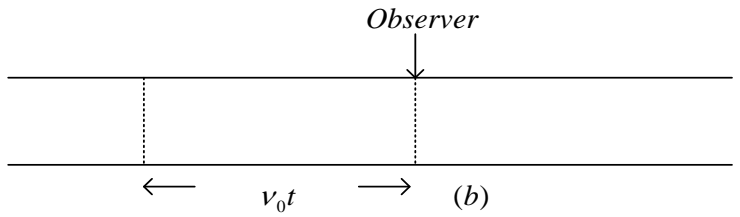

Figure .1 (a) Constant Flow of Cars

(b) Distance Traveled in Hours for a Single Car $t$

\subsection{Conservation Law}

The models for traffic, whether they are one-equation or system of equations, are based on the physical principle of conservation. When physical quantities remain the same during some processes, these quantities are said to be conserved. Putting this principle into a mathematical representation will make it possible to predict the densities and velocities patterns at future time. In our case, the number of cars in a segment of a highway $\left[x_{1}, x_{2}\right]$ is our physical quantities, and the process is to keep them fixed (i.e., the number of cars coming in equals the number of cars going out of the segment). Consider a stretch of highway on which cars are moving from left to right as shown in Figure 2. It is assumed here that there are no exit or entrance ramps. The number of cars within $\left[x_{1}, x_{2}\right]$ at a given time $t$ is the integral of the traffic density given by

$$
N=\int_{x_{1}}^{x_{2}} \rho(x, t) d x .
$$

The number of cars can still change (increase or decrease) in time due to cars crossing both ends of the segment. Assuming no cars are crated or destroyed, and then the change of the number of cars is due to the change at the boundaries only. Therefore, the rate of change of the number of cars is given by

$$
\frac{d N}{d t}=f_{\text {in }}(\rho, v)-f_{\text {out }}(\rho, v) \text {, }
$$

since the number of cars unit time is the flow $f(\rho, v)$. Combining Equations 7 , and 8 , yields the integral conservation law

$$
\frac{d}{d t} \int_{x_{1}}^{x_{2}} \rho(x, t) d x=f_{\text {in }}(\rho, v)-f_{\text {out }}(\rho, v) .
$$


This equation represents the fact that change in number of cars is due to the flows at the boundaries. Let the end points are independent variables (not fixed with time), then the fulll derivative is replaced by partial derivative to get

$$
\frac{\partial}{\partial t} \int_{x_{1}}^{x_{2}} \rho(x, t) d x=f_{\text {in }}(\rho, v)-f_{\text {out }}(\rho, v) .
$$

The change in the number of cars with respect to distance is given by

$$
f_{\text {in }}(\rho, v)-f_{\text {out }}(\rho, v)=-\int_{x_{1}}^{x_{2}} \frac{\partial f}{\partial x}(\rho, v) d x
$$

and by setting the last two equations equal to each other, we get

$$
\int_{x_{1}}^{x_{2}}\left[\frac{\partial \rho}{\partial t}(x, t)+\frac{\partial f}{\partial x}(\rho, v)\right] d x=0
$$

This equation states that the definite integral of some quantity is always zero for all values of the independent varying limits of the integral. The only function with this feature is the zero function. Therefore, assuming $\rho(x, t)$ and $f(x, t)$ are both smooth, the one-dimensional conservation law is found to be

$$
\frac{\partial}{\partial t} \rho(x, t)+\frac{\partial}{\partial x} f(\rho, v)=0 .
$$

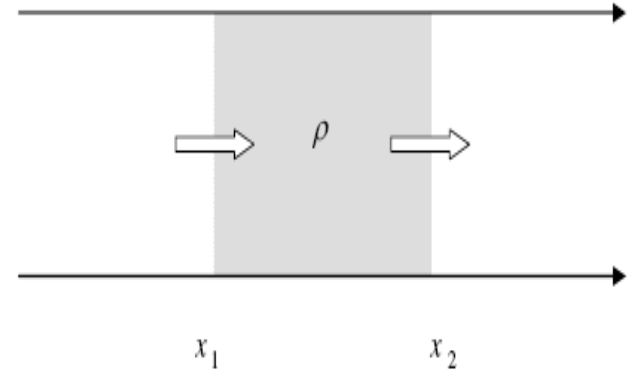

Figure 2. One Dimension Flow

\subsection{Weak Solutions}

The function $\rho$ is needed to interpret as a solution in a weak sense of the initial-value problem Equation 14. In particular, assume we use method of characteristics to see that even for smooth initial conditions the strong solutions cannot be extended in time indefinitely. In fact, even smooth initial conditions can lead to discontinuous solutions in finite time. In this case, it can be used test function $\phi \in C_{0}^{1}(\mathfrak{R} \times \mathfrak{R})$. $C_{0}^{1}$ is the space function that are continuously differentiable with compact support. $\phi(x, t)$ is identically zero outside of some bounded set, and so the support of the function lies in a compact set.

$$
\left.\begin{array}{l}
\phi: \mathfrak{R} \times[0, \infty) \rightarrow \mathfrak{R}, \\
\phi \in C_{0}^{\infty}(\mathfrak{R} \times[0, \infty)),
\end{array}\right\}
$$

by parts and we call $\phi$ is a test function. Then multiply the equation $\rho_{t}+f(\rho)_{x}=0$ by $\phi$ and integrate:

$$
\begin{aligned}
& \left.\int_{0}^{+\infty} \int_{-\infty}^{+\infty}\left(\rho_{t}+f(\rho)_{x}\right) \phi d x d t\right|_{t=0}=0 \\
& -\int_{0}^{+\infty} \int_{0}^{+\infty} \rho \phi_{t} d x d t-\left.\int_{-\infty}^{+\infty} \rho \phi d x\right|_{t=0}=0 \\
& -\int_{0}^{+\infty} \int_{-\infty}^{+\infty} f(\rho) \phi_{x} d x d t=0 .
\end{aligned}
$$

Using the initial condition $\rho(x, 0)=\rho_{0}(x)$ on

$\mathfrak{R} \times\{t=0\}$, we have

$$
\int_{0}^{+\infty} \int_{-\infty}^{+\infty} \rho \phi_{t}+f(\rho) \varphi_{x} d x d t+\int_{-\infty}^{+\infty} \rho_{0} \phi(x, 0) d x=0
$$

\subsection{Scalar Riemann Problem}

Scalar Riemann problem is the Cauchy problem for the scalar conservation law where the initial data is a piecewise constant function with only two values. In both cases there will be two different values on both sides of $x=0$ at time $t=0$. In one case the left hand side value will be lower in the other it will be higher than the right hand side value.

The initial value problem

$$
\begin{aligned}
& \frac{\partial}{\partial t} \rho(x, t)+f^{\prime}(\rho) \frac{\partial}{\partial x} \rho=0, \\
& \rho(x, 0)=\rho_{0}
\end{aligned}
$$

with the piecewise constant initial function

$$
\rho(x, 0)= \begin{cases}\rho_{l}, & \text { if } x<0, \\ \rho_{r}, & \text { if } x>0,\end{cases}
$$

is called Riemann's problem for the scalar conservation law. $\rho_{r}, \rho_{l} \in \mathfrak{R}$ are the left and right initial states, with $\rho_{l} \neq \rho_{r}$.

(i) If $\rho_{l}>\rho_{r}$, from the lax entropy condition of the for convex fluxes, the unique entropy solution of the Riemann problem is

$$
\rho(x, t)= \begin{cases}\rho_{l}, & \text { if } x<s t, \\ \rho_{r}, & \text { if } x>s t,\end{cases}
$$

where $x \in \mathfrak{R}, t>0$ and $s$ is given by the RankineHugoniot condition; 
$s=\frac{\left(\rho_{l}+\rho_{r}\right)}{2}$

is the shock speed, the speed at which the discontinuity travels

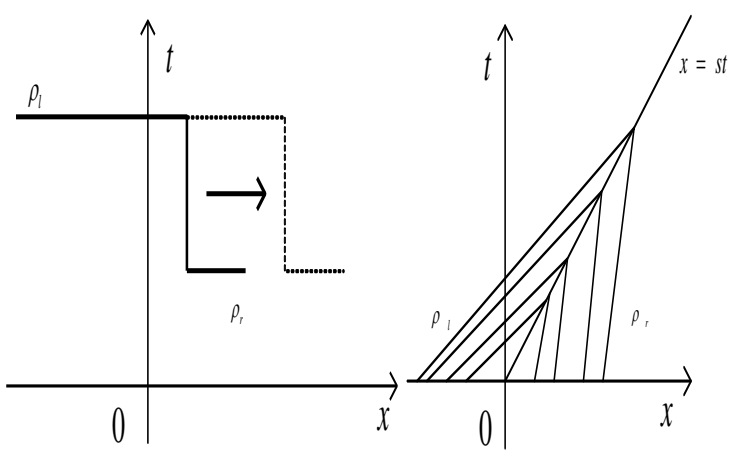

Figure 3. Shock Wave

(ii) If $\rho_{r}>\rho_{l}$, the unique entropy solution of the Riemann's problem

$$
\rho(x, t)= \begin{cases}\rho_{l} & \text { if } \frac{x}{t}<f^{\prime}\left(\rho_{l}\right), \\ \left(f^{\prime}\right)^{-1}\left(\frac{x}{t}\right) & \text { if } f^{\prime}\left(\rho_{l}\right)<\frac{x}{t}<f^{\prime}\left(\rho_{r}\right), \\ \rho_{r} & \text { if } \frac{x}{t}>f^{\prime}\left(\rho_{r}\right),\end{cases}
$$

where $x \in R, t>0$.

(iii) If $\rho_{l}=\rho_{r}$, there exists a unique entropy solution of the Riemann problem, that is the constant state $\rho(x, t)=\rho_{l}=\rho_{r}$.

Therefore in each case we can give an explicit expression of the solution of the Riemann's problem.

In the first case the two constant states $\rho_{l}$ and $\rho_{r}$ are separated by a shock wave with constant speed $s$. In the other case the two states are separated by a rarefaction wave. The rarefaction solution is continuous in $\mathfrak{R} \times(0, \infty)$. From a physical point of view it corresponds to expensive states of fluids and it is commonly known as the rarefaction wave.

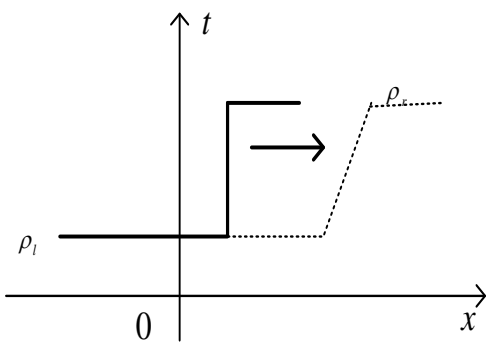

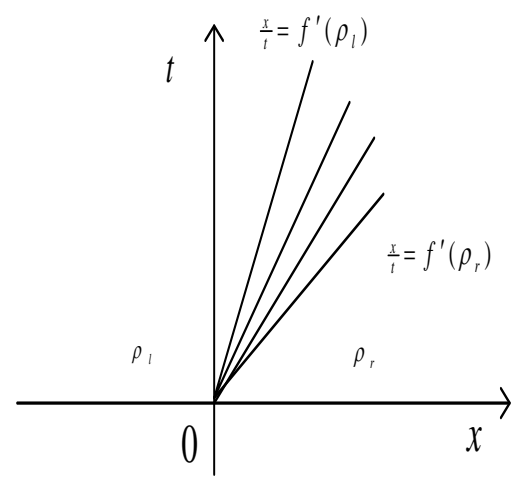

Figure 4. Rarefaction Wave

\subsection{Shock Speed}

The propagating shock Equation 18 is a weak solution to Burgers' Equation only if the speed of propagation is given by Equation 17. The same discontinuity propagating at a different speed would not be a weak solution. The speed of propagation can be determined by conservation. If $M$ is large compared to $s t$ then by Equation.10,

$\int_{-}^{M} \rho(x, t) d x$ must increase at the rate

$$
\begin{aligned}
\int_{-M}^{M} \rho(x, t) d x & =f\left(\rho_{l}\right)-f\left(\rho_{r}\right) \\
& =\frac{1}{2}\left(\rho_{l}+\rho_{r}\right)\left(\rho_{l}-\rho_{r}\right)
\end{aligned}
$$

$$
\begin{aligned}
\int_{-M}^{M} \rho(x, t) d x & =f\left(\rho_{l}\right)-f\left(\rho_{r}\right) \\
& =\frac{1}{2}\left(\rho_{l}+\rho_{r}\right)\left(\rho_{l}-\rho_{r}\right)
\end{aligned}
$$

for Burger's equation. On the other hand, the Equation 16 has

$\int_{-M}^{M} \rho(x, t) d x=(M+s t) \rho_{l}+(M-s t) \rho_{r}$

so that

$\frac{d}{d t} \int_{-M}^{M} \rho(x, t) d x=s\left(\rho_{l}-\rho_{r}\right)$.

For arbitrary flux function $f(\rho)$ this same argument gives the following relation between the shock speed $S$ the states $\rho_{l}$ and $\rho_{r}$, called the Rankine- Hugoniot $(R-H)$ jump condition:

$f\left(\rho_{r}\right)-f\left(\rho_{l}\right)=s\left(\rho_{r}-\rho_{l}\right)$.

For a scalar conservation law the shock speed is

$$
s=\frac{f\left(\rho_{l}\right)-f\left(\rho_{r}\right)}{\rho_{l}-\rho_{r}}
$$




$$
=\frac{[f]}{[\rho]}
$$

where [.] indicates the jump in some quantity across the discontinuity. Note that any jump is allowed, provided the speed is related Equation 24.

For systems of equations, $\rho_{l}-\rho_{r} \quad$ and $f\left(\rho_{r}\right)-f\left(\rho_{l}\right)$ are both vectors while $s$ is still a scalar. We cannot always solve for $S$ to make Equation 23 holds. Only certain jumps $\rho_{l}-\rho_{r}$ are allowed, those for which the vectors $f\left(\rho_{r}\right)-f\left(\rho_{l}\right)$ and $\rho_{l}-\rho_{r}$ are linearly dependent.

For a linear system

$$
f\left(\rho_{r}\right)-f\left(\rho_{l}\right)=s\left(\rho_{r}-\rho_{l}\right),
$$

$\rho_{l}-\rho_{r}$ must be an eigenvector of the matrix and $S$ is the associated eigenvalue. For a linear system, these eigenvalues are the characteristic speeds of the system. Thus discontinuities can propagate only along characteristics, a fact that we have seen for the scalar case.

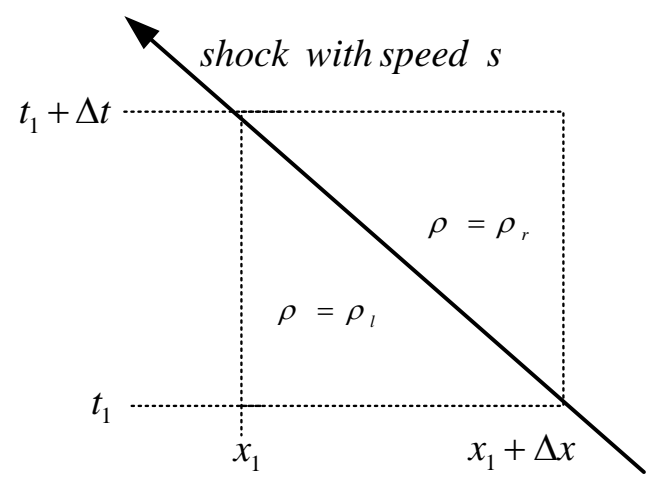

Figure 5. Region of Integration for Shock Speed Calculation

\subsection{Vehicle Path}

The velocity of vehicles is independent of traffic wave velocity. Once a vehicle a distance $D$ encounters traffic wave which moves with velocity $-v_{\max }$, the car will begin to move. The density in the expansion fan is

$$
\rho(x, t)=\rho_{\max }\left(\frac{v_{\max } t-x}{2 v_{\max } t}\right) .
$$

The velocity of a car is $v=v_{\max }\left(1-\frac{\rho}{\rho_{\max }}\right)$.

The path $x(t)$ of the car thus satisfies

$$
\frac{d x}{d t}=v_{\max }\left(1-\frac{1}{\rho_{\max }}\left[\rho_{\max }\left(\frac{v_{\max } t-x}{2 v_{\max } t}\right)\right]\right) .
$$

\section{CALCULATED RESULT FOR SHOCK SPEED}

Let $f=60 \rho\left(1-\frac{\rho}{300}\right)$ in miles/ hours units. We

suppose that $t=0$ the density on road is given by

$\rho(x, 0)= \begin{cases}40, & \text { for } x<0 \\ 40(1+3 x), & \text { for } 0<x<1 \\ 160, & \text { for } x>1 .\end{cases}$

Since $v(\rho)=60\left(1-\frac{\rho}{300}\right)$, the characteristics are

$x= \begin{cases}30 t+x_{0}, & \text { for } x_{0}<0, \\ 60\left(\frac{2}{3}-x_{0}\right) t+x_{0}, & \text { for } 0<x_{0}<1, \\ -10 t+x_{0} & , \quad \text { for } x_{0}>1 .\end{cases}$

In the transition region characteristics are thus given by $x=60\left(\frac{2}{3}-x_{0}\right) t+x_{0}$. At time $t=\frac{1}{60}$, these characteristics intersect at $x=\frac{2}{3}$ mile, and a shock forms. The shock velocity is given by

$$
\begin{aligned}
v_{\text {shock }} & =\frac{d \xi}{d t}=\frac{\left[f\left(\rho_{l}\right)-f\left(\rho_{r}\right)\right]}{\left[\rho_{r}-\rho_{l}\right]} \\
& =60(1-(40+160) / 300) \\
& =20 \mathrm{mph} .
\end{aligned}
$$

Consider what happens to the car at $x=-1$ when $t=0$.

The speed of the car is initially

$60(1-40 / 300)=52 m p h . \quad$ At $\quad t=\frac{1}{60}, \quad$ it has reach $x=0$ and the shock forms at. $x=\frac{2}{3}$. Since the car's path is given by $x_{c a r}=40 t-1$ and the shock path by $x_{\text {shock }}=20(t-1 / 60)+2 / 3$ the car will meet the shock when $x_{\text {car }}=x_{\text {shock }}$ or $t=\frac{1}{15}$ hours. Once through 


$$
\begin{aligned}
& \text { the shock, the car moves at velocity } \\
& 60=\left(1-\frac{160}{300}\right)=28 m p h .
\end{aligned}
$$

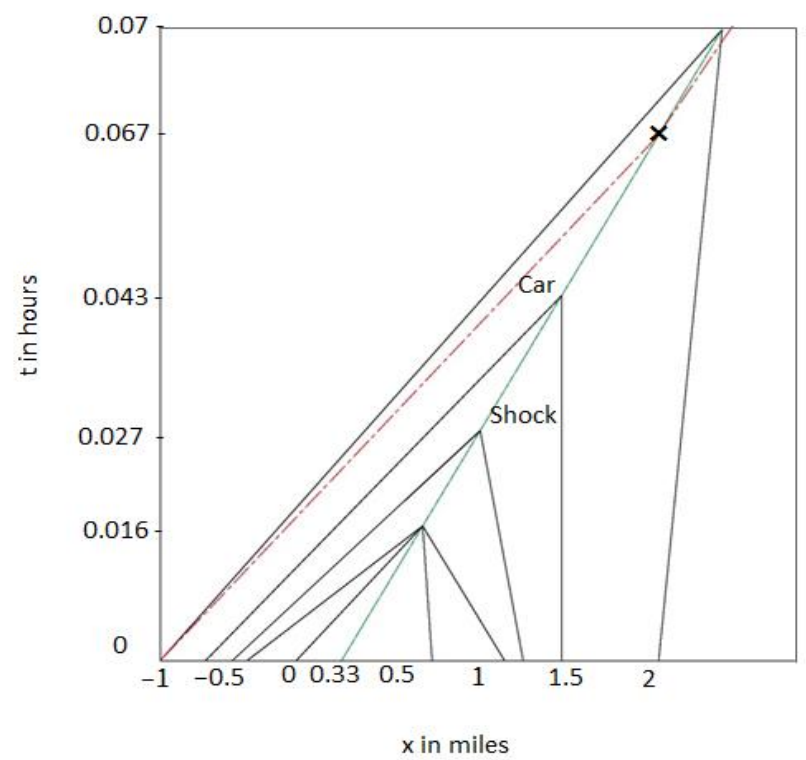

Figure 6. Shock Formation from the Initial Density

\section{CONCLUSIONS}

In this paper, we focused on road networks, where on each road the scalar conservation laws model and car following models determines the evolution of the car traffic. Then we calculated for the shock speed.

\section{ACKNOWLEDGMENT}

The authors would like to acknowledge the support of the papers for their references.

\section{REFERENCES}

[1] Kac, P.: Opdtimal and Feedback Control for Hyperbolic Conservation Laws, Faculty of the Virginia Polytechnic Institute and States University, Virginia, (2007)

[2] Bretti, G., and Piccoli, B.: Traffic Flow on Road Networks, SIAM Journal on Mathematical Analysis 36, (2005) pp. 1862- 1886

[3] Leveque, R.J.: Finite Volume Methods for Hyperbolic Problems, Cambridge Texts in Applied Mathematics, Cambridge University Press, (2002).

[4] Piccoli, B.: Numerical Approximations of a Traffic Flow Models on Networks, Networks and Heterogeneous Media, No. 1, (206) pp. 57-84.

[5] Klar. A., and Rascle, M.: Derivation of Continuum Flow Traffic Models from Microscopic Follow the Leader Models, SIAM J. Appl. Math., (2002) pp. 259.

[6] Randall J. Le Veque: Numerical Methods for Conservation Laws, Second Edition. 